\title{
Astro Tourism sites in Georgia
}

\author{
Nino Kochiashvili* \\ E.Kharadze Georgian National Astrophysical Observatory, Mt.Kanobili, Abastumani, 0301, Adigeni, Georgia
}

\begin{abstract}
The main astro site in Georgia is the Abastumani Astrophysical Observatory. The history of astronomy in Georgia begins no later than IX century. During the Middle Ages, there were at least four observatories in Georgia, including the Arab Observatory in Tbilisi (Narikala - a fortress). This observatory operated between IX-XIV centuries. Two other observatories functioned in monasteries in eastern part and one - in western part of the country. All these places are active touristic sites in Georgia.
\end{abstract}

Keywords: Astronomy, History of Astronomy, Astro Tourism

\section{The Abastumani Observatory}

The Abastumani Observatory, or E. Kharadze Georgian National Astrophysical Observatory was founded in 1932. This is a "classical" observatory with small telescopes. Observations are carried out in almost all fields of astronomy. We observe variable stars, the Sun, the bodies of the Solar system and extragalactic objects - Blazars and other AGNs. Observations for optical counterparts of GRBs and GW sources added to the above list in recent years.

Usually the observatory hosted only schools for excursions and only on weekends. The observatory is open for everyone every day from 11 am to 1 am starting from 1990es. Tourists have the opportunity to look at interesting celestial objects using the 40-cm refactor, visit the museum of the Georgian astronomy and attend popular lectures. Visitors come from different parts of Georgia, as well as from different countries.

\section{Middle Ages}

An arabian observatory was established in Tbilisi in the IX century. The observatory was situated close to the Narikala fortress and functioned until XIV century. It seems that the observatory had rather qualified staff. For example, when Nasir Al-Din Al-Tusi began to build Maragheh observatory according to Hulagu Khan's order, he invited Fakhrt Al-Din Al-Ikhlati from the Tbilisi observatory together with Mu'ayyad Al-Din Al Urdi, Fakhr Al-din Al Maghrabi and Najm AL-Din Al-Qazwini. There are some archaeological findings on the site of the observatory that are especially important because "we know practically nothing about the buildings themselves and, by the way, our ignorance is not restricted to this particular observatory (Maragheh observatory - N. K.); we may say that in general we hardly know anything about the organization of Greek and Muslim observatories" Sarton (1929).

Gelati Academy in the western part and Ikalto Academy in the eastern part of Georgia were established in the beginning of the XII century. They have been active for several centuries and Astronomy was one of the mandatory subjects there. It is known that observatories existed in these academies, and Georgian kings and nobles were observing themselves for astrological purposes. David the Builder the king who reigned in 1089-1125, wrote himself in his "The canon of repentance" about observing celestial objects Jokhadze (2005).

*nino@abao.ge 
It is highly likely that observatories existed also in other monasteries of Georgia, including the David Gareja complex.

All these places are active tourist destinations nowadays.

\section{References}

Jokhadze L., 2005, David the Builder, The Canon of Rependence. Ilia State University, Kakutsa Cholokashvili ave. 3/5, 0162, Tbilisi, Georgia

Sarton G., 1929, Isis, 13, 111 